\title{
HIDROCARBONETOS POLICÍCLICOS AROMÁTICOS (HPAs) EM AGUARDENTES
}

\author{
S. M. BETTIN ; D. Wagner FRANCO
}

\section{RESUMO}

A presença de hidrocarbonetos aromáticos polinucleares (HPAs) em aguardentes foi investigada por cromatografia líquida (CLAE) após sua prévia extração em fase sólida (SPE). A separação foi realizada em uma coluna Supelco, LCPAH-octadecil silano (25cm x $4,6 \mathrm{~mm} \times 5 \mu \mathrm{m}$ ) com gradiente acetonitrila/água e a quantificação utilizando detector de fluorescência. Os HPAs (naftaleno; acenaftaleno; fluoreno; fenantreno; antraceno; fluoranteno; pireno; 1,2- benzo(e)pireno; criseno; benzo(e)pireno; 2,3-benzo(a)antraceno; 1,2-benzo(b)fluoranteno; benzo(k)fluoranteno; dibenzo(a,h)antraceno; benzo(a)pireno; benzo(ghi)pirileno foram identificados e quantificados em vinte e oito amostras de aguardentes de cana. Os resultados experimentais para as amostras de aguardentes (cachaças) são analisados em termos de análises de componentes principais (PCA) objetivando a diferenciação entre o perfil das aguardentes produzidas a partir de cana-de-açúcar queimada e não-queimada.

Palavras-chave: aguardente de cana; hidrocarboneto aromático polinucleares; cana queimada.

\section{SUMMARY}

PHAs IN SPIRITS. The presence of PHAs (polycyclic aromatic hydrocabons) in spirits has been investigated using high performance liquid chromatography (HPLC) after solid phase extraction (SPE). The separation was achieved with a Supelco LCPAH-octadecil silane column [ $25 \mathrm{~cm} \times 4,6 \mathrm{~mm} \times 5 \mu \mathrm{m}]$ and acetonitrile/water elution gradient and the quantification using a fluorescence detector. The PHAs (naphthalene; acenaphthene; fluorene; phenantrene; anthracene; phuorantene; pyrene; 1,2benzo(e)pyrene; chrysene; benzo(e)pyrene; 2,3-benzo(a)anthracene; 1,2-benzo(b)phluoranthene; benzo(k)fluoranthene; dibenzo(a,h)anthracene; benzo(a)pyreno; benzo(ghi)pyrilene were quantifed in twenty eight samples of sugar cane spirits. All the experimental data for sugar cane spirit have been analyzed through principal components analysis (PCA) aiming to compare the chemical profile of beverages produced from burned and not burned sugar cane.

Keywords: sugar cane spirit; burned sugar cane; polycyclic aromatic hydrocarborns.

\section{1 - INTRODUÇÃO}

Os hidrocarbonetos aromáticos polinucleares (HPAs) são originados por fontes antropogênicas (queima de florestas, atividades industriais e atividades residenciais) ou por atividades naturais (atividades vulcânicas e biosintese por algas) $[4,12,13]$. Estima-se que, de 12 a 14 toneladas de benzo(a)pireno são lançados anualmente na atmosfera pelos vulcões $[4,12]$.

Até o começo do século XX havia um equilíbrio entre a produção e a degradação natural de HPAs sendo a sua concentração baixa e constante. Com o aumento do desenvolvimento industrial, esse balanço natural foi perturbado e a razão entre a produção e a degradação de HPAs tem aumentado constantemente $[4,12]$. O mecanismo de formação de HPAs durante a combustão incompleta de material orgânico é conhecido há muito tempo [12]. Acredita-se que estejam envolvidos dois processos distintos: a pirólise e a pirossintese. Em altas temperaturas, compostos orgânicos são convertidos em moléculas pequenas não estáveis (pirólise). Estas e outros ra-

Recebido para publicação em 21/08/2002. Aceito para publicação em 02/03/2005 (000957).

Departamento de Quimica e Física Molecular, Instituto de Química de São Carlos, Universidade de São Paulo (USP). End.: Av. Trabalhador São Carlense, n. 400. CEP: 13560-970, São Carlos, SP. E-mail:douglas@iqsc.sc.usp.br

A quem a correspondência deve ser enviada. dicais se recombinam para produzir moléculas maiores e mais estáveis de HPAs (pirossintese). Uma vez formados os HPAs podem sofrer reações pirossintéticas, originando estruturas mais complexas com anéis altamente condensados [12]. Os HPAs não são obrigatoriamente fracionados em fragmentos menores antes da pirossintese, podendo resistir á fragmentação parcial seguida pela hidrogenação dos seus radicais primários. Em geral, todos os compostos orgânicos contendo carbono e hidrogênio, podem servir como precursores de HPAs [4, 12].

Estudos na Holanda demonstraram que a ingestão de açúcar puro e produtos açucarados são fontes importantes de HPAs na dieta da população. As análises destes produtos apresentaram teores de criseno de até $36 \mu \mathrm{g} / \mathrm{kg}$ [13]. As análises de amostras de açúcares comerciais brasileiros indicaram a presença de HPAs em concentrações que variam de 0,25 a $0,83 \mu \mathrm{g} / \mathrm{kg}$ [11].

Na Alemanha "The German Society for Fat Science" fixou para o teor total de HPAs em alimentos o limite de $25 \mu \mathrm{g} / \mathrm{L}$ e de $5 \mu \mathrm{g} / \mathrm{L}$ para o teor de HPAs de alta massa molecular (1,2-benzo(b)fluoranteno, benzo(k)fluoranteno, benzo(a)pireno, benzo(ghi)pirileno e benzo(a)antraceno) $[9,10]$.

A existência de HPAs em bebidas não é novidade. Foi constatada a presença de benzopireno, benzofluoranteno e criseno em uísques (atribuida a queima da turfa) em

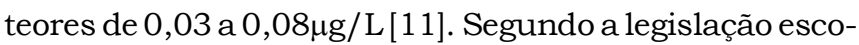
cesa as concentrações máximas toleradas de HPAs em alimentos são de $1 \mu \mathrm{g} / \mathrm{L}$ para o naftaleno; acenafteno; fluoreno; fenatreno; antraceno e de $2 \mu \mathrm{g} / \mathrm{L}$ para o fluorante- 
no; pireno e criseno [5].

Além da queima da cana, a presença de HPAs em bebidas pode ser proveniente de contaminações nas etapas do processo de produção, tais como: o emprego de lubrificantes nos equipamentos; a utilização de recipientes não-adequados para o armazenamento da bebida; tanques revestidos com resinas asfálticas ricas em HPAs; pelo açúcar adicionado na bebida e durante a maturação da bebida em tonéis de madeira tratada termicamente [1, 7, 14].

A colheita da cana-de-açúcar no Brasil (que ocorre todo ano entre maio e novembro) e em outros países é conduzida geralmente após a queima dos canaviais [14]. Este procedimento primitivo resulta primeiramente em contaminação ambiental, pois partículas de palha queimada (fuligem), bem como inúmeros compostos tóxicos dentre os quais destacamos os HPAs são introduzidos na atmosfera. Estudos realizados em amostras de fuligem da cana-de-açúcar queimada indicam a presença dos seguintes HPAs: naftaleno, acenaftaleno, acenaftileno, fluoreno, fenantreno, antraceno, fluoranteno, pireno, 1,2benzo(e)pireno, benzo(a)antraceno, criseno, benzo(e)pireno, 1,2-benzo(b)fluoranteno, benzo(k)fluoranteno, benzo(a)pireno e benzo(ghi)pirileno [14].

Apesar de bebidas contaminadas por hidrocarbonetos policíclicos aromáticos (HPAs) serem potencialmente prejudiciais à saúde, pois benzo(a)pireno e criseno são compostos considerados carcinogênicos [2, 5, 6], no Brasil até o momento não existe legislação específica sobre o assunto.

O objetivo deste trabalho é contribuir para o conhecimento do perfil químico das aguardentes brasileiras com relação ao seu conteúdo de HPAs. Para tanto é apresentada uma adaptação da metodologia existente [14] para a análise de HPAs e uma tentativa de diferenciar aguardentes provenientes de cana queimada e nãoqueimada pela aplicação do método de análise de componentes principais (PCA) aos resultados obtidos.

\section{2 - MATERIAL E MÉTODOS}

\section{1 - Amostras}

As aguardentes analisadas foram: A.A.A (SP), Armazem Vieira (SP)", Azuladinha (AL), Beija Flor (SP) 1996, Beija Flor (SP) 1998 Beija Flor (SP) 1999 , Bosco(SP)", Catedral (SP) , 51 (SP) , Colosso (SP) , Havana (MG) ", Izê (SP)", Jamel (SP)", Lua Cheia (SP) , Lua Nova (SP) ${ }^{\circ}$, Mangueira (PI) , Paraty (RJ) ${ }^{b}$, Pirassununga (SP) , Pitú Gold (CE) , Santa Ângela (1998), Santa Ângela (1999) , São Francisco (RJ) ', Terra Brasilis (SP), Tiquara (SP) , Trinca 3 (CE) , Velho Barreiro (SP)", Vila Velha (SP)", Ypioca prata $(\mathrm{CE})$.

Esta classificação é arbitrária, não estando relacionada com as propriedades organolépticas das bebidas nem com sua aceitação comercial. Foram coletadas amostras de aguardentes de várias regiões do país, considerando-se a tradição de cada região na produção de cachaça.

Por meio de contato com produtores foi possivel classificar as bebidas como provenientes de cana queimada e não-queimada.

\section{2 - Reagentes}

Os padrões utilizados para as análises dos compostos fenólicos foram todos de grau analítico (Merck e Aldrich) e estão listados a seguir: naftaleno, acenaftaleno, fluoreno, fenantreno, antraceno, fluoranteno, pireno, 1,2- benzo(e)pireno, criseno, benzo(e)pireno, 2,3benzo(a)antraceno, 1,2-benzo(b)fluoranteno, benzo(k)fluoranteno, 9,10-dimetil benzo(a) antraceno, benzo(a)pireno e benzo(ghi)pirileno. A escolha dos padrões foi efetuada considerando os compostos previamente identificados [14] em amostras de outros destilados. Os solventes utilizados foram metanol, isopropanol, acetonitrila e acetato de etilade sempre de grau HPLC (Merck, Carlo Erba e Aldrich).

\section{3 - Extração}

A extração dos HPAs das amostras foi realizada pela técnica SPE (extração em fase sólida), utilizando os cartuchos ENVI-LC, (octadecil silano) Supelco, 6,0mL. Foram testadas diversas fases de eluição para os HPAs

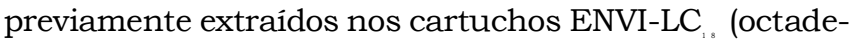
cil silano) Supelco. O acetato de etila foi o solvente que apresentou os melhores resultados para a eluição dos HPAs de massa molecular maior, o isopropanol para os HPAs de massa molecular menor (naftaleno, acenaftaleno, fluoreno, fenantreno) (Tabela 2). Cada cartucho foi ativado com 10,0mL metanol. Aliquotas de 12,0mL de amostra foram aplicadas nos cartuchos e eluídas com $2,0 \mathrm{~mL}$ de isopropanol e $2,0 \mathrm{~mL}$ de acetato de etila $(1: 1 \mathrm{v} / \mathrm{v})$. O extrato obtido foi concentrado por evaporação com ar sintético e o volume final acertado para $1,00 \mathrm{~mL}$, utilizando para tanto acetato de etila.

\section{4 - Análise cromatográfica}

As análises dos HPAs foram realizadas utilizando os seguintes equipamentos e condições experimentais: Cromatógrafo Líquido: Shimadzu modelo LC-10AD; detector: fluorescência, modelo RF-551; coluna: LC-PAH (octadecil silano) $25 \mathrm{~cm}$ x $2,1 \mathrm{~mm}$ x $5 \mu \mathrm{m}$; fluxo: 0,40mL/min. Gradiente de eluição: A:água/ B:acetonitrila;

Programação de comprimento de onda:

$\begin{array}{ccc}\text { Tempo (min) } & \lambda_{\mathrm{ex}},_{\mathrm{n} m}: & \lambda_{\mathrm{e} m},_{\mathrm{nm}}: \\ 0 & 275 & 320 \\ 15 & 265 & 380 \\ 24 & 290 & 395 \\ 39 & 290 & 406\end{array}$




\section{5 - Quantificação}

A curva de calibração foi construída, a partir da adição de volumes conhecidos de solução estoque contendo $1000 \mathrm{mg} / \mathrm{L}$ dos HPAs em solução de isopropanol e acetato de etila $(1: 1 \mathrm{v} / \mathrm{v})$ em aguardente isenta de HPAs (teores abaixo dos limites de detecção). Estas soluções foram preparadas de forma a obter padrões nas seguintes concentrações: $0,50 \mathrm{mg} / \mathrm{L}, 0,25 \mathrm{mg} / \mathrm{L}, 0,10 \mathrm{mg} / \mathrm{L}, 0,05 \mathrm{mg} / \mathrm{L} \mathrm{e}$ $0,02 \mathrm{mg} / \mathrm{L}$. O padrão de criseno devido à baixa solubilidade foi preparado da seguinte forma: pesou-se uma solução super-saturada do padrão. O resíduo foi filtrado, seco, pesado e a concentração final da solução foi calculada subtraindo da massa inicialmente pesada a massa não solubilizada.

\subsection{Limite de detecção (LD) e quantificação (LQ) e reprodutibilidade do método.}

O limite de detecção foi calculado na prática como sendo correspondente à concentração que produzira um valor do sinal medido três vezes maior que o nivel do ruído. O nivel ruído (linha de base) foi calculado a partir de uma corrida do branco [3, 8].

Considerou-se o limite de quantificação como sendo o menor valor determinado, com confiabilidade aceitável de precisão e exatidão, para uma dada condição analitica. Em geral, um valor bastante aceitável para LQ é o de 3,3 x LD, ou seja, menor quantidade calculada é considerada como sendo três vezes maior que o minimo detectável em um dado sistema e dez vezes maior que o nivel do ruído. A reprodutibilidade do método para os compostos estudados foi determinada realizando-se cinco medidas da mesma amostra durante dois dias, sempre em duplicata $[3,8]$.

\section{3 - RESULTADOS E DISCUSSÃO}

As análises dos HPAs foram realizadas em uma coluna Supelco-LC-PAH ( $25 \mathrm{~cm} \times 2,1 \mathrm{~mm} \times 5 \mu \mathrm{m})$ (octadecil silano), a qual apresentou uma boa separação para uma solução contendo os padrões de HPAs e para uma amostra de aguardente de cana (Figuras 1 e 2).

As Tabelas 1 e 2 reúnem as características da metodologia empregada. A Tabela 1 apresenta os valores de reprodutibilidade para o método utilizado cujo desvio padrão relativo varia entre $0,0002 \%$ a $0,44 \%$ para o fenatreno e o criseno, respectivamente. O limite de detecção varia entre $5,0 \times 10^{-3} \mu \mathrm{g} / \mathrm{L}$ (antraceno) a $0,10 \mu \mathrm{g} / \mathrm{L}$ para 9,10-dimetil-1,2-benzoantraceno. O limite de quantificação está entre $0,01 \mu \mathrm{g} / \mathrm{L}$ (antraceno, benzo(a)pireno, dibenzo(a,h)antraceno) e 1,0 $\mathrm{g} / \mathrm{L}$ para o naftaleno.

Os valores de reprodutibilidade (Tabela 1) para o método utilizado em termos do desvio padrão relativo variam entre 0,0002\% (fenatreno) a 0,44\% (criseno), o limite de detecção varia entre $5,0 \times 10^{-3} \mu \mathrm{g} / \mathrm{L}$ (antraceno) a $0,10 \mu / L$ (naftaleno).

A Tabela 2 apresenta os resultados de recuperação
TABELA 1 - Testes de reprodutibilidade para a metodologia utilizada para a analise de HPAs

\begin{tabular}{lcccc}
\hline \multicolumn{1}{c}{ HPAs } & Média $(\mu \mathrm{g} / \mathrm{L})$ & DPR $(\%)^{*}$ & $\begin{array}{c}\text { Limite de deteção } \\
(\mu \mathrm{g} / \mathrm{L})\end{array}$ & $\begin{array}{c}\text { Limite de quantificação } \\
(\mu \mathrm{g} / \mathrm{L})\end{array}$ \\
\hline Naftaleno & 0,17 & 0,02 & 0,10 & 1,00 \\
Acenaftaleno & 0,26 & $6 \times 10^{3}$ & 0,010 & 0,20 \\
Fluoreno & 0,02 & 0,40 & 0,01 & 0,10 \\
Fenantreno & 0,29 & $2 \times 10^{-4}$ & 0,01 & 0,10 \\
Antraceno & 0,08 & 0,06 & $5 \times 10^{-3}$ & 0,01 \\
Fluoranteno & 0,07 & 0,06 & 0,010 & 0,20 \\
Pireno & 0,70 & $1 \times 10^{-3}$ & 0,010 & 0,04 \\
Benzo(a)antraceno & 0,08 & 0,06 & 0,010 & 0,10 \\
Criseno & 0,02 & 0,44 & 0,001 & 0,10 \\
Benzo(e)pireno & 0,18 & 0,01 & 0,010 & 0,30 \\
9,10-dibenzopireno & 0,80 & $6 \times 10^{-3}$ & 0,010 & 0,25 \\
Benzo(b)fluoranteno & 0,07 & 0,07 & 0,020 & 0,20 \\
Benzo(k)fluoranteno & 0,05 & 0,08 & 0,010 & 0,10 \\
Dibenzo(a,h)antraceno & 0,19 & 0,01 & 0,001 & 0,01 \\
Benzo(a)pireno & 0,08 & 0,05 & 0,001 & 0,01 \\
Benzo(ghi)pirileno & 0,09 & 0,01 & 0,001 & 0,10 \\
\hline
\end{tabular}

TABELA 2 - Testes de recuperação da extração dos HPAs com mistura de isopropanol: acetato de etila (1:1/v:v)

\begin{tabular}{lc}
\hline \multicolumn{1}{c}{ HPAs } & Recuperação (\%) \\
\hline Acenaftaleno & $99,3 \pm 1,50$ \\
Fluoreno & $100,4 \pm 8,0$ \\
Fenantreno & $98,5 \pm 5,60$ \\
Antraceno & $97,5 \pm 4,60$ \\
Fluoranteno & $98,5 \pm 4,20$ \\
Pireno & $88,1 \pm 3,70$ \\
Benzo(a)antraceno & $100,9 \pm 4,50$ \\
Criseno & $87,2 \pm 3,30$ \\
Benzo(e)pireno & $101,5 \pm 4,80$ \\
9,10-dibenzopireno & $82,0 \pm 2,20$ \\
Benzo(b)fluoranteno & $99,3 \pm 4,60$ \\
Benzo(k)fluoranteno & $99,4 \pm 1,50$ \\
Dibenzo(a,h)antraceno & $98,1 \pm 6,90$ \\
Benzo(a)pireno & $99,4 \pm 4,20$ \\
Benzo(ghi)pirileno & $89,6 \pm 5,60$ \\
\hline
\end{tabular}

da extração para os HPAs com a mistura da solução de isopropanol e acetato de etila $(1: 1 \mathrm{v} / \mathrm{v})$. Estes valores variam de $89,6 \%$ (benzo(ghi)pireleno) a 101,5\% (benzo(e)pireno).

A Tabela 3 reúne os resultados experimentais para os teores de HPAs em aguardentes produzidas com cana queimada e não-queimada. Os teores médios de HPAs, isolados e totais, para aguardentes produzidas com cana queimada são sempre bem superiores em relação às aguardentes produzidas com cana não-queimada. Os HPAs: fluoreno, fenantreno, antraceno, fluoranteno, pireno, benzo(a)antraceno, criseno e benzo(e)pireno, não foram detectados em aguardentes produzidas com cana não-queimada.

As aguardentes tipo "b" e "c" produzidas com cana queimada, apresentam teores $(10,60 \mu / \mathrm{L}$ e $11,80 \mu / \mathrm{L})$ médios acima do recomendado $(1,0 \mu \mathrm{g} / \mathrm{L})$ na legislação esco- 
TABELA 3 - Teores médios de HAPs em bebidas $* *(\mu \mathrm{g} / \mathrm{L})$

\begin{tabular}{lccc}
\hline \multicolumn{1}{c}{ HAPs } & $\begin{array}{c}\text { Teor } \\
\text { Limite }^{*}\end{array}$ & Aguardente (cana não-queimada) & $\begin{array}{c}\text { Aguardente } \\
\text { (cana queimada) }\end{array}$ \\
\hline aftaleno & 1.00 & 6.00 & 9.70 \\
cenaftaleno & 1,00 & 2,00 & 9,30 \\
luoreno & 1.00 & 0.50 & 3.45 \\
enantreno & 1.00 & 0.28 & 2.75 \\
ntraceno & 1,00 & $\mathrm{ND}$ & 3,40 \\
luoranteno & 2,00 & 0,70 & 2,91 \\
ireno & 2.00 & $\mathrm{ND}$ & 3.47 \\
enzo(a)antraceno & 2,00 & $\mathrm{ND}$ & 1,07 \\
riseno & 2,00 & $\mathrm{ND}$ & 1.10 \\
enzo(e)pireno & 2.00 & $\mathrm{ND}$ & 1.86 \\
enzo(b)fluoranteno & 2,00 & 0,25 & 2,50 \\
enzo(k)fluoranteno & 2,00 & 0,40 & 3,70 \\
ribenzo(a.h)antraceno & 2.00 & 0.05 & 1.45 \\
enzo(a)pireno & 2,00 & $\mathrm{ND}$ & 1,55 \\
enzo(ghi)pirileno & 2.00 & 0.86 & 4.50 \\
\hline
\end{tabular}

* limite recomendado na legislação escocesa; ND: valores abaixo dos limites de detecção da Tabela 1

** valor médio calculado com base no resultado de 28 amostras.

cesa para o naftaleno. As aguardentes tipo "b" e "c" apresentam também, teores $(8,52 \mu \mathrm{g} / \mathrm{L}, 3,10 \mu / \mathrm{L}$ e 4,42 e $3,94 \mu / \mathrm{L}$, respectivamente) acima do recomendado $(1,0 \mu \mathrm{g} / \mathrm{L})$ para o acenafteno e o fluoreno. Os teores médios em aguardentes tipo "a" apresentam teores $(5,45 \mu \mathrm{g} / \mathrm{L}$, $4,88 \mu \mathrm{g} / \mathrm{L}$ e $10,03 \mu \mathrm{g} / \mathrm{L})$ acima que o recomendado $(2,0 \mu \mathrm{g} / \mathrm{L})$ para o fluoranteno, benzo(k)fluoranteno e benzo(a)pireno. As aguardentes tipo "b" apresentam teores $(3,47 \mu \mathrm{g} / \mathrm{L} \mathrm{e} 3,43 \mu \mathrm{g} / \mathrm{L})$ acima do recomendado $(2,0 \mu \mathrm{g} / \mathrm{L})$ para o pireno e o benzo(a)antraceno.

A avaliação dos resultados, segundo as análises das componentes principais (PCA), foi utilizada no intuito de verificar a existência ou não de diferenças entre os perfis

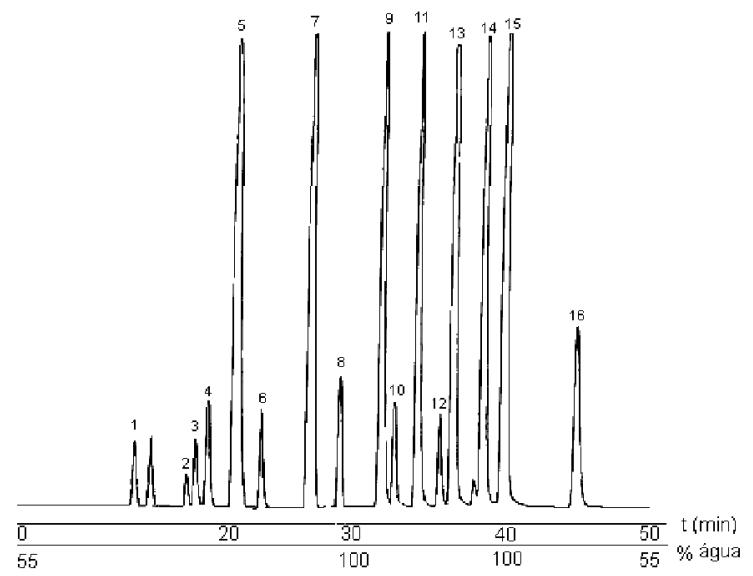

FIGURA 1 - Cromatograma dos padrões de HPAs em coluna Supelco-LC-PAH $(25 \mathrm{~cm} \times 4,6 \mathrm{~mm}$ x $5 \mu \mathrm{m})(1,0 \mu \mathrm{g} / \mathrm{L})$, (octadecil silano) (HPLC; detector de fluorescência; volume de injeção; 1-naftaleno; 2-acenaftaleno; 3-fluoreno; 4-fenantreno; 5antraceno; 6-fluoranteno; 7-pireno; 8-(1,2-benzo(e)pireno); 9-criseno; 10-benzo(e)pireno; 11-(2,3-benzo(a)antraceno); 12-(1,2-benzo(b)fluoranteno); 13-benzo(k)fluoranteno; 14(9,10-dimetil benzo(a) antraceno); 15-benzo(a)pireno; 16benzo(ghi)pirileno

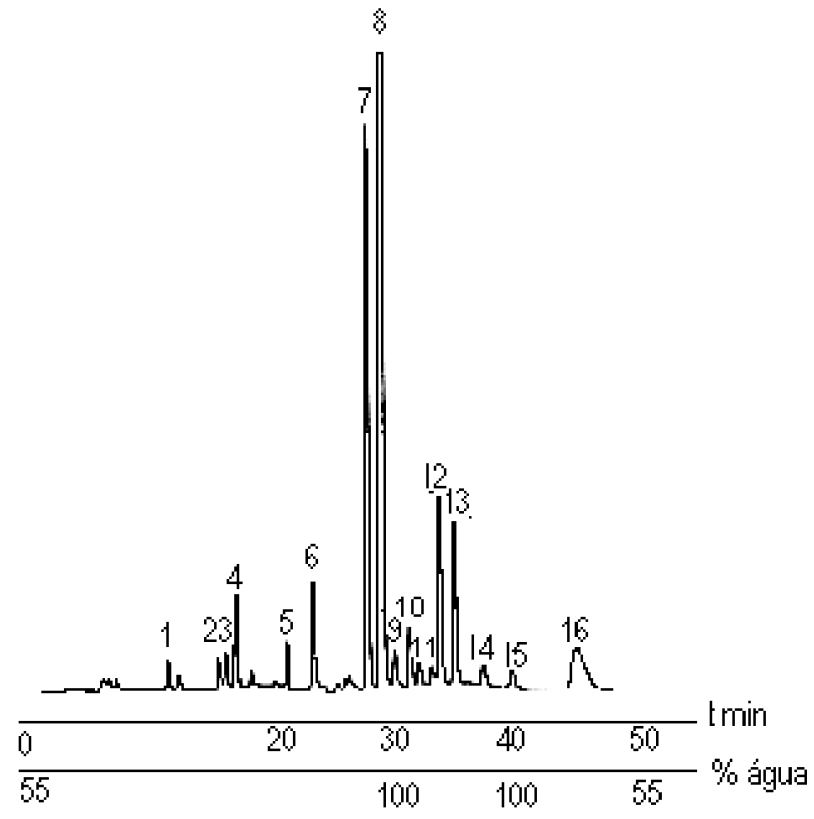

FIGURA 2 - Cromatograma típico da aguardente de cana (HPLC;; detector de fluorescência; coluna LC-PAH (octadecil silano); volume de injeção de $25 \mu \mathrm{L}$. 1-naftaleno; 2acenaftaleno; 3-fluoreno; 4-fenantreno; 5-antraceno; 6fluoranteno; 7-pireno; 8-(1,2-benzo(e)pireno); 9-criseno; 10benzo(e)pireno; 11-(2,3-benzo(a)antraceno); 12-(1,2benzo(b)fluoranteno); 13-benzo(k)fluoranteno; 14dibenzo(a,h)antraceno; 15 -benzo(a)pireno; 16 benzo(ghi)pirileno

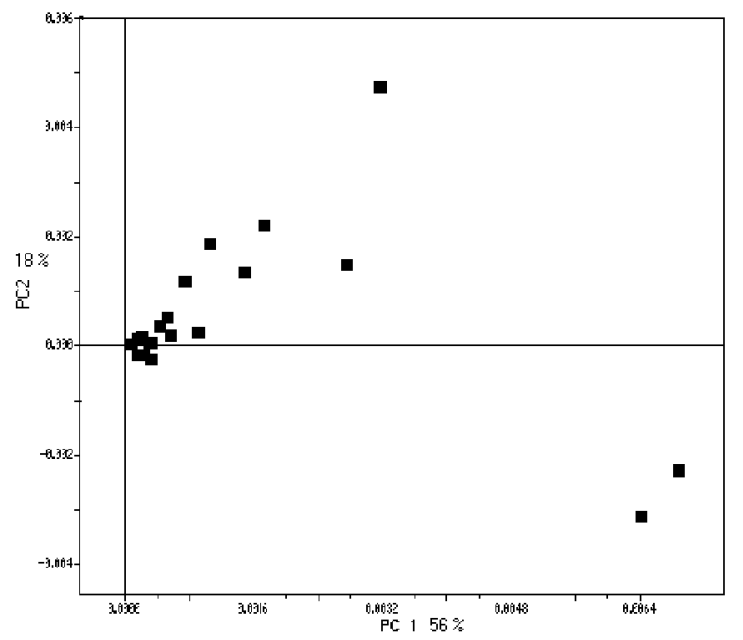

FIGURA 3 - PC1 x PC2 para aguardentes produzidas com cana queimada (azul; n: 16) e aguardentes produzidas com cana não queimada (vermelho; n: 9) 


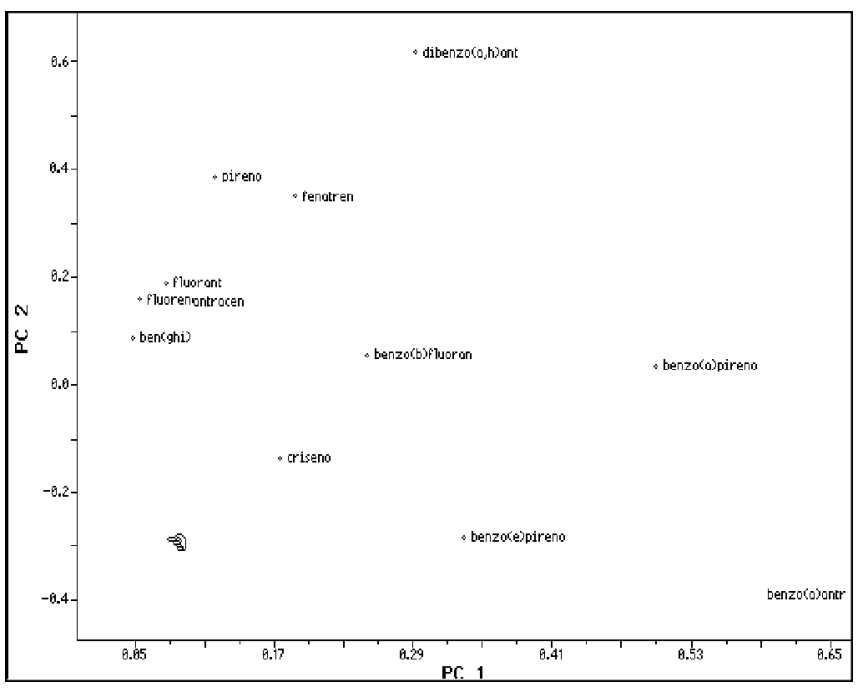

FIGURA 4 - PC1 (56\%) x PC2 (18\%) para aguardentes produzidas com cana queimada e aguardentes produzidas com cana não-queimada

de aguardentes produzidas com cana queimada e nãoqueimada. A Figura 3 sugere ser possivel discriminar as aguardentes produzidas com cana queimada e nãoqueimada, com base nos teores de HPAs. O benzo(a)pireno, benzo(b)pireno, o benzo(a)antraceno e o benzo(e)pireno são os HPAs que mais contribuem para as aguardentes produzidas com cana queimada (Figura 4).

Estes resultados indicam o efeito da queima da cana-de-açúcar sobre os teores de HPAs das aguardentes e sugerem a possibilidade de discriminar o produto de acordo com a procedência da cana.

\section{4 - CONCLUSÕES}

A metodologia adaptada apresenta boa reprodutibilidade, exatidão e sensibilidade permitindo a análise de 16 HPAs em aguardente de cana. É de simples aplicação reunindo características que a recomendam para o controle de rotina. A análise dos resultados indicou a presença de HPAs, em concentrações variáveis nas amostras. Nas aguardentes provenientes de cana nãoqueimada não foi detectada a presença de antraceno, pireno, benzo(a)antraceno, criseno, benzo(e)pireno e benzo(a)pireno. É promisor o tratamento de dados analíticos de teores de HPAs por métodos quimiométricos (PCA) para verificar a qualidade da cana utilizada na produção da aguardente.

\section{5 - REFERÊNCIAS BIBLIOGRÁFICAS}

[1] AKHLAQ, M. S. Detailed Analysis of crude oil group types using reversed-phase high-performance liquid chromatography. Journal of Chromatography, v. 677, p.265-272, 1994.

[2] CAMARGO M. S.; TOLEDO, M. C., Hidrocarbonetos Aromáticos Policíclicos em Margarina, Creme Vegetal e
Maionese, Ciênc. Tecnol. Aliment., Campinas, n. 20, p. 51-55, 2000.

[3] CAUSON, R. Validation of Chromatographic Methods in Biomedical Analysis. Viewpoint and Discussion. Journal of Chromatography B, v.689, p. 175-180, 1997.

[4] CRESSER, M.; KILlHAM, K.; EDWARDS T. Soil Chemistry and its Applications, Cambridge, New YorK, 1993, 322p.

[5] DENNIS, M.J.; MASSEY, R.C. Analysis of polycyclic hydrocarbons in UK total diets. Food Chemistry Toxicology, v. 21, p. 569-574, 1983.

[6] FÁlCON, M. S. G. Enchainment of Benzo(a)pyrene in smoke food products and determination by highperformance liquid-chromatography-fluorescence detection. Journal of Chromatography A, vol. 753, p.107-121, 1994.

[7] HAWTHORNE, S.B.; TREMBLEy, S.; MONIOT, C.B. Static subcritical water extration with simultaneous solid-phase extraction for determining polycyclic aromatic hydrocarbons on environmental solids. Journal of Chromatography A, v. 886, p. 237-244, 2000.

[8] JENKE, D.R. Chromatography method validation: a review of current practices and procedures II. Guidelines for primary validation parameters. Journal of Liquid Chromatography, v. 19, n.5, p.719-736, 1996.

[9] MORET, S., Determination of Polyclic Aromatic Hydrocarbons in Water and Water-based alcoholic Beverages. Zeischrift fur Lebensmittel, v. 201, n.4, p.322-326, 1995.

[10] MORET, S.; CONTE, L. Polycyclic aromatic hydrocarbons in edible fats and oils: occurrence and analytical methods. Journal of Chromatography $\mathbf{A}, \mathrm{v}$. 882, p.245-253, 2000.

[11] NOVAES, V. F. Hidrocarbonetos em Aguardente de Cana, Escola Superior de Agricultura Luiz de Queiroz, Departamento de Ciência e Tecnologia AgroindustrialUniversidade de São Paulo (artigo não publicado).

[12] TREVELIN, W R. Otimização da Análise de Hidrocarbonetos Policíclicos Aromáticos em sistemas aquosos. São Carlos, 1992, p.83, Dissertação (Mestrado)-Istituto de Química de São Carlos, Universidade de São Paulo.

[13] VOS, R. H.; DOKKUM, W. Polycyclic Aromatic Hydrocarbons in total diet samples. Food Chemical Toxicology, v. 28, p. 263-268, 1990.

[14] ZAMPERLINNI, C.M.G., Silva-Santiago, M., Vilegas, W. Solid-phase extration of sugar cane soot extract for analysis by gas chromatography with flame ionization and mass spectrometric detection. Journal of Chromatography A, v. 889, p. 281-289, 2000.

\section{6 - AGRADECIMENTOS}

Os autores agradecem à Fundação de Amparo à Pesquisa do Estado de São Paulo (FAPESP) pelo apoio financeiro. 